\title{
Effect of local heterogeneous conditions on growth curves of $P$-wave
}

\author{
Kyosuke OKAMOTO and Seiji TSUNO ${ }^{1}$ \\ ${ }^{1}$ Center for Railway Earthquake Engineering Research, Railway Technical Research Institute
}

\begin{abstract}
It has been adopted empirically that a growth rate of initial P-waves decreases as the epicentral distances become distant. Using this relationship, epicentral distances are estimated from the growth curve of initial P-waves immediately after the occurrence of earthquake in the current Earthquake Early Warning (EEW) system. However the growth rates are not only the function of epicentral distances but also the function of seismic source function, heterogeneous condition of medium, etc. So the growth rates of different earthquakes are fluctuated each other. This fluctuation decreases the accuracy of the EEW. In this study we reveal that a main cause of the fluctuation is seismic scattering and the fluctuation has locality. Finally we propose a robust procedure to estimate epicentral distances considering the locality.
\end{abstract}

\section{Introduction}

According to Odaka (2003), epicentral distances can be estimated from the envelope curve of initial P-waves. In the current Earthquake Early Waning (EEW) system in Japan, initial estimation of epicentral location is done within few seconds after the P-wave arrival. To estimate epicentral distance, equation 1 has been proposed by Yamamoto et al. (2012).

$$
C t
$$

where, $t$ represents time. Equation 1 is fitted to growing curve of initial P-waves. From the $C$ value, epicentral distances are estimated using a relationship, which is the $C$ value decreases as the epicentral distance increases mainly due to geometrical spreading and anelastic attenuation (Figure 1). However in practice, the $C$ values of different earthquakes having the same epicentral distance show quiet different values. The gap in the $C$ values is sometimes by several hundred times and



Figure $1 C$ value against epicentral distance. The observation term is from 1997 to 2011. The solid line is an approximation curve. it makes about $10^{2} \mathrm{~km}$ order error in the estimated epicentral distance.

In this study we hypothesize that a major cause of the gap in the $C$ values is derived from difference in heterogeneous condition in subsurface. Seismic waves attenuate and scattered according to the regional heterogeneities. Due to these effects, the $C$ value fluctuates from the predicted value. To verify it, we theoretically calculate P-waves in heterogeneous medium considering the conversion waves between $\mathrm{P}$ - and S-waves. We show that variation in condition of heterogeneities, in this study we focus on correlation distance, makes the $C$ value fluctuate significantly. Then we compare the theoretically calculated $C$ values with one calculated from earthquakes in 1996 - 2011 observed by the K-NET and KiK-net. We calculate a regional parameter of heterogeneities (correlation distance) in Japan which can account the fluctuation in the observed $C$ values. As a result, we find that the spatial distribution of the obtained correlation distance show locality.

In this study we hypothesize that variation in the condition of heterogeneities makes the $C$ value fluctuate significantly and verify it. If we treat the fluctuation in the $C$ values by considering the local condition of heterogeneities, estimation on epicentral distances becomes more accurate. We propose a method to improve the accuracy of estimation of epicentral distances.

\section{Theoretical calculation of $C$ value}

We theoretically calculate seismic waves propagating in heterogeneous medium based on the Born approximation according to Sato (1984). 


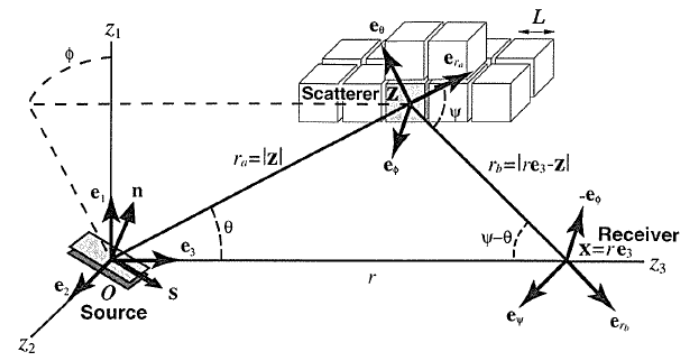

Figure 2 Calculation field (reprinted from Yoshimoto, 1997a)

Figure 2 is geometry of the calculation, in which power spectral density of PP PS and SP conversions (equations 2 -4) are calculated.

$$
\begin{aligned}
& S_{i}^{P P}=\frac{\widehat{W}^{P}(\omega)}{4 \pi \rho_{0} \alpha_{0}} \int_{-\infty-\infty) \infty}^{\infty} \int_{-\infty}^{\infty} d \mathbf{z} \delta\left(t-\frac{r_{a}}{\alpha_{0}}-\frac{r_{b}}{\alpha_{0}}\right) \frac{1}{r_{a}^{2} r_{b}^{2}} B_{r}^{P}(\theta, \phi ; \mathbf{n}, \mathbf{s})^{2} \\
& \times\left\{\frac{\left\langle\left\langle\left.{ }^{T} F_{r}^{P P}(\psi, \zeta ; \omega)\right|^{2}\right\rangle\right.}{L^{3}}\right\}\left(\mathbf{e}_{r_{b}} \cdot \mathbf{e}_{i}\right)^{2} \exp \left\{-Q_{P}^{-1}(\omega) \omega t\right\} \\
& S_{i}^{P S}=\frac{\widehat{W}^{P}(\omega)}{4 \pi \rho_{0} \alpha_{0}} \int_{-\infty-\infty)-\infty}^{\infty} \int_{-\infty}^{\infty} d \mathbf{z} \delta\left(t-\frac{r_{a}}{\alpha_{0}}-\frac{r_{b}}{\beta_{0}}\right) \frac{1}{r_{a}^{2} r_{b}^{2}} B_{r}^{P}(\theta, \phi ; \mathbf{n}, \mathbf{s})^{2} \\
& \times\left\{\frac{\left\langle\left|F_{\psi}^{P S}(\psi, \pi ; \omega)\right|^{2}\right\rangle}{L^{3}}\right\}\left(\mathbf{e}_{\psi} \cdot \mathbf{e}_{i}\right)^{2} \exp \left\{-\frac{Q_{P}^{-1}(\omega) \omega r_{a}}{\alpha_{0}}-\frac{Q_{S}^{-1}(\omega) \omega r_{b}}{\beta_{0}}\right\} \\
& S_{i}^{S P}=\frac{\hat{W}^{S}(\omega)}{4 \pi \rho_{0} \beta_{0}} \int_{-\infty-\infty}^{\infty} \int_{-\infty-\infty}^{\infty} d \mathbf{z} \delta\left(t-\frac{r_{a}}{\beta_{0}}-\frac{r_{b}}{\alpha_{0}}\right) \frac{1}{r_{a}^{2} r_{b}^{2}} B_{\theta}^{S}(\theta, \phi ; \mathbf{n}, \mathbf{s})^{2} \\
& \times\left\{\frac{\left\langle\left. F_{r}^{S P}(\psi, \pi ; \omega)\right|^{2}\right\rangle}{L^{3}}\right\}\left(\mathbf{e}_{r_{b}} \cdot \mathbf{e}_{i}\right)^{2} \exp \left\{-\frac{Q_{S}^{-1}(\omega) \omega r_{a}}{\beta_{0}}-\frac{Q_{P}^{-1}(\omega) \omega r_{b}}{\alpha_{0}}\right\}
\end{aligned}
$$

where, $\widehat{W}^{P}$ and $\widehat{W}^{S}$ are spectral density of radiated energy from the fault. $\alpha, \beta$ and $\rho$ are $\mathrm{P}$ - and S-waves velocities and density. $B$ is radiation parameter and $F$ is scattering amplitude. $Q_{p}$ and $Q_{S}$ show attenuation parameters of $\mathrm{P}$ - and $\mathrm{S}$-waves. $\omega$ is angular frequency and $\mathbf{z}$ is position vector. The source is double couple and $\mathbf{n}$ is normal vector of the fault and $\mathbf{s}$ is direction of the slip. The Power spectral densities are calculated for $4-20 \mathrm{~Hz}$ with 1 $\mathrm{Hz}$ intervals. Acceleration wave form is obtained by equation 5 .

$$
\sqrt{\left\langle\left|\dot{v}_{i}(\mathbf{x}, t)\right|^{2}\right\rangle_{T}}=2 \sqrt{2 \sum_{n} \omega_{n}^{2}\left\langle S_{i}\left(\mathbf{x}, t ; 2 \pi f_{n}\right)\right\rangle \Delta f_{n}}
$$

where, $f$ is frequency and $<>$ denotes the ensemble average. Heterogeneous condition is characterized by autocorrelation of fluctuation of elastic parameters $R$. In this study, $R$ is expressed by the Exponential Auto Correlation Function (ACF, equation 6).

$$
R(r)=\varepsilon^{2} \exp \left(-\frac{r}{a}\right)
$$

where, $\varepsilon$ is fluctuation magnitude and $a$ is correlation distance. $r$ shows distance.

Figure 3 shows theoretical envelope for the earthquake occurred on May, 8, 2005 in Tochigi

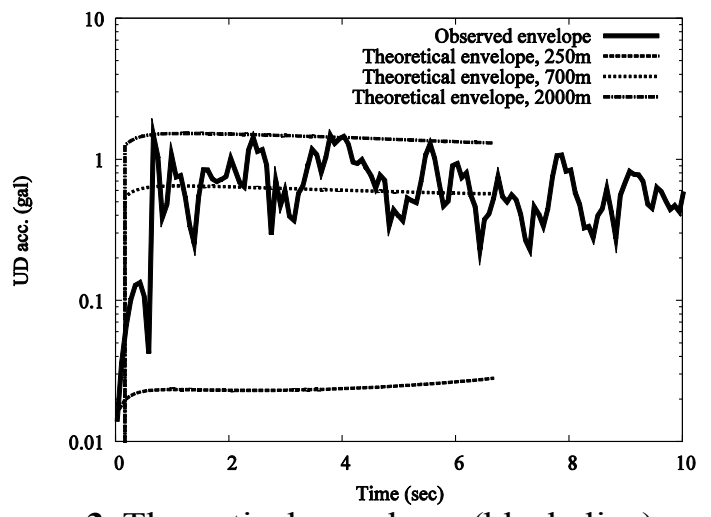

Figure 3 Theoretical envelope (black line) and observed envelope (red line). We set $\varepsilon$ to $8 \%$ and $a$ to 250,700 or $2000 \mathrm{~m}$ (semilogarithmic graph).

prefecture. The magnitude $\left(M_{j}\right)$ is 4.5 and depth is $9.7 \mathrm{~km}$. The theoretical envelope of $a=700 \mathrm{~m}$ agrees well with the observed one. By fitting equation 1 to the envelopes, theoretical and observed $C$ values are obtained. $0.5 \mathrm{sec}$ long envelope from P-wave arrival is used. In this case, the theoretical $C\left(C_{\text {theo. }}\right)$ values are $5.34 \times 10^{-2}(a=250 \mathrm{~m}), 1.47(a=700 \mathrm{~m})$ and $3.45(a=2000 \mathrm{~m})$ respectively while the observed $C$ $\left(C_{\text {obs. }}\right)$ value is 1.20 . The range of $a$ value agrees with that reported by Yoshimoto (1997b). $C$ value is strongly fluctuated by the value of $a$.

\section{Real data analysis}

Earthquakes $\left(M_{j}>4.0\right)$ observed by K-NET and KiK-net during March 1996 to April, 2011 are used. We call the whole earthquake as "data set".

\section{(1) Determination of regional $a$ value}

In the section 2 , we revealed that $C$ value fluctuates by difference in $a$ value. In this chapter, we reveal that $a$ value shows locality by comparing $C_{o b s .}$ value with $C_{\text {theo. }}$ value.

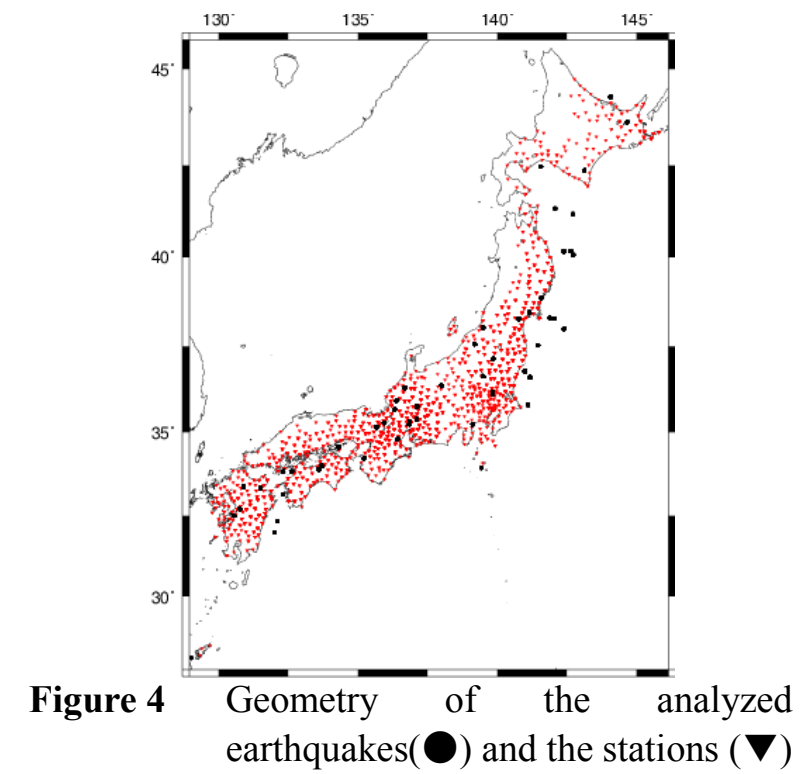


The earthquakes whose magnitude is from 4.0 to 5.0 from the data set are used for the analysis (geometry is shown in Figure 4). Total number of the events is 55 and that of observed seismograms is 2,373 . At each seismic station, $a$ value is chosen to satisfy equation 7 .

$$
\min _{a} \sum_{\text {event }} C_{\text {obs }} / C_{\text {theo. }}
$$

In the calculation of $C_{\text {theo. }}$, $\mathrm{P}$ - and $\mathrm{S}$-wave velocities (Matsubara and Obara, 2011) at the intermediate point of the source and the receiver are used. To simplify, we chose $a$ value from $100 \mathrm{~m}, 150 \mathrm{~m}, 200 \mathrm{~m}$, $300 \mathrm{~m}, 400 \mathrm{~m}$ and $600 \mathrm{~m}$. $\varepsilon$ is fixed to $6 \%$. As a result, it is found that $a$ shows longer value around the Pacific side of Kanto and Tohoku regions. Shorter $a$ value is seen around Itoigawa-Shizuoka Tectonic Line and San'in region (Figure 5). The tendency agrees with the spatial distribution of coda- $Q\left(Q_{c}\right)$ reported by Carcole and Sato (2010). The short $a$ value areas mean that there are strong heterogeneities. These areas correspond with the low $Q_{c}$ value area, that is, highly attenuated medium. The long $a$ value areas also correspond with high $Q_{c}$ value area.
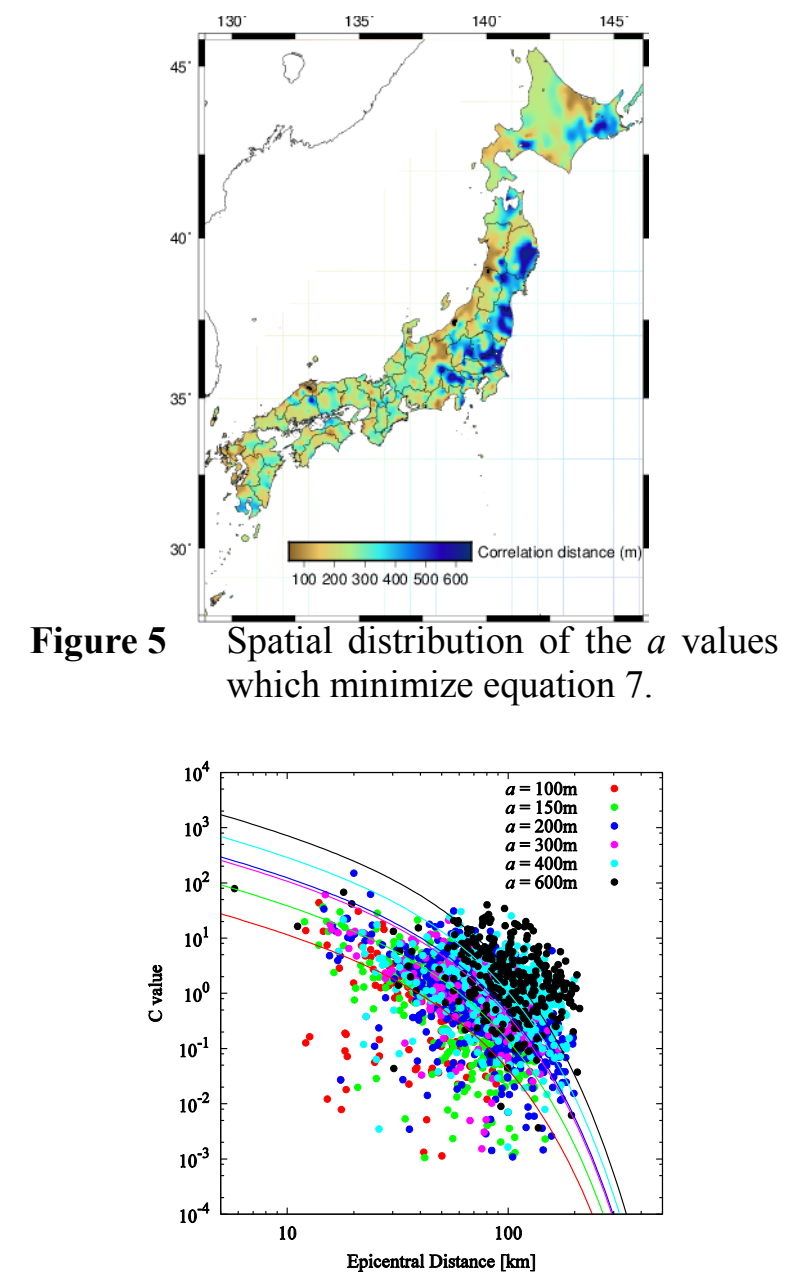

Figure $6 C_{o b s .}$ values classified in terms of $a$ values of the stations and approximation curves.
Figure 6 shows $C_{o b s .}$ values classified in terms of the $a$ value of the stations. $C_{o b s .}$ values obtained at the stations of shorter $a$ are smaller. When the medium is strongly heterogeneous (short $a$ ), initial $\mathrm{P}$-waves are strongly scattered and attenuated, thus, the energy in the initial $\mathrm{P}$-waves is redistributed to following wave trains and absorbed. It makes the growing curve of initial $\mathrm{P}$-waves gradual and consequently small $C_{o b s}$. values are observed. In the areas of longer $a$ values, conversely, high $C_{o b s}$. values are observed due to sharp growing curves.

\section{(2) Improvement of epicentral estimation}

Epicentral distance $\Delta$ are estimated from $C$ value using equation 8 .

$$
\log C=\chi \log \Delta+\eta+\kappa \cdot \Delta
$$

where, $\chi$ involves geometrical spreading, $\eta$ locality and $\kappa$ scattering and intrinsic attenuation. When these parameters are determined using the whole dataset (the values are $\chi=-1.319, \eta=2.748, \kappa=$ $\left.-4.011 \times 10^{-3}\right)$, the estimated epicentral distance includes error to some extent. The error sometimes reaches $10^{2} \mathrm{~km}$ order (see Figure 1). In this study, we propose a new procedure to estimate epicentral distances. The parameters in equation 8 are determined at each seismic station according to the value of $a$. In this study, the seismic stations are divided into six groups, that is, $a$ is $100 \mathrm{~m}, 150 \mathrm{~m}$, $200 \mathrm{~m}, 300 \mathrm{~m}, 400 \mathrm{~m}$ or $600 \mathrm{~m}$. Theoretically $\chi$, which involves geometrical spreading, is -1.0 for body wave and -0.5 for surface wave. Through the six groups, we set $\chi$ to -1.0 . Values of $\eta$ and $\kappa$ are needed to be determined for the respective groups. However, for simplicity, we use the same value of $\kappa$ for the six groups and determine values of $\eta$ for each group by a grid search method. The obtained approximation curves are shown in Figure 6. Values of $\chi, \eta$ and $\kappa$ are in Table 1.

The longer $a$ value is, the higher $\eta$ value is. When $\eta$ value becomes higher, the approximation curve is elevated entirely (see Figure 6). By this elevation, regional differences in $C_{o b s}$. value are taken account. This result is obtained by analyzing the earthquakes of $M_{j} 4.0-5.0$ from the dataset. So that, we consider that the effect of source function

Table 1 Values of equation 8 for each groups

\begin{tabular}{c|ccc}
\hline$a$ & $\chi$ & $\eta$ & $\kappa$ \\
\hline $100 \mathrm{~m}$ & -1.00 & 2.22 & $-1.60 \times 10^{-2}$ \\
$150 \mathrm{~m}$ & -1.00 & 2.74 & $-1.60 \times 10^{-2}$ \\
$200 \mathrm{~m}$ & -1.00 & 3.25 & $-1.60 \times 10^{-2}$ \\
$300 \mathrm{~m}$ & -1.00 & 3.19 & $-1.60 \times 10^{-2}$ \\
$400 \mathrm{~m}$ & -1.00 & 3.62 & $-1.60 \times 10^{-2}$ \\
$600 \mathrm{~m}$ & -1.00 & 4.02 & $-1.60 \times 10^{-2}$ \\
\hline
\end{tabular}



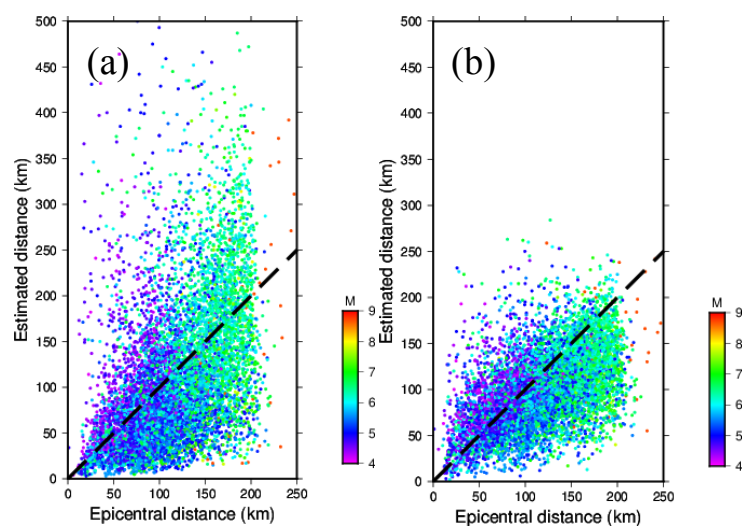

Figure 7 The horizontal axis shows the epicentral distance from JMA and the vertical axis shows the estimated epicentral distance. (a) The case of the current method. (b) The case of our proposed method.

on initial P-waves can be negligible because the corresponding source duration time $(\mathrm{sec})$ is $10^{-2}-$ $10^{-1}$ order while the time window we used is $0.5 \mathrm{sec}$. In this condition, local heterogeneities affect the $C_{\text {obs. }}$ value mainly.

Figure 7 shows the estimated epicentral distance using equation 8 against the actual epicentral distance. When the parameters in equation 8 are estimated using the whole dataset (current method), error in the estimated epicentral distance is sometimes reaches $10^{2} \mathrm{~km}$ order (Figure 7a). On the other hand, when the parameters in equation 8 are determined for the groups (classified by $a$ value) respectively, the estimation error decreases well. The parameters are determined using the earthquakes of $M_{j} 4.0-5.0$. However, epicentral distances are well estimated for the earthquakes larger than $M_{j}$ 5.0.

\section{Summary}

In this study, we hypothesized that $C$ values for a given epicentral distance widely spreads due to difference in local heterogeneous condition and verified it using the theoretical calculation. Then using the theoretical calculation, we revealed the regional $a$ value distribution. Finally we showed that accuracy of estimation of epicentral distances improves by changing the parameters of the approximation equation according to region's $a$ value.

ACKNOWLEDGMENT: We would like to thank the National Research Institute for Earth Science and Disaster Prevention (NIED) to make available $\mathrm{K}$ - and KiK-net data and the Japan Meteorological Agency (JMA) to make available JMA unified earthquake catalog. Some of the figures were drawn by Generic Mapping Tools (GMT) software (Wessel and Smith 1995).

\section{REFERENCES}

1) Carcole E, and Haruo S, 2010, Spatial distribution of scattering loss and intrinsic absorption of short-period $\mathrm{S}$ waves in the lithosphere of Japan on the basis of the Multiple Lapse Time Window Analysis of Hi-net data, Geophys. J. Int., 180, 268-290.

2) Matsubara M, and Obara K, 2011, The 2011 off the Pacific coast of Tohoku Earthquake related to a strong velocity gradient with the Pacific plate, Earth Planet Space, 63, 663-667.

3) Odaka T, Ashiya K, Tsukada S, Sato S, Ohtake K, and Nozaka D, 2003, A new method of quickly estimating epicentral distance and magnitude from a single seismic record, Bull. Seismol. Soc. Am., 93, 526-532.

4) Sato H, 1984, Attenuation and envelope formation of three-component seismograms of small local earthquakes in randomly inhomogeneous lithosphere, J. Geophys. Res., 89, 1221-1241.

5) Wessel P, amd Smith WHF, 1995, New version of generic mapping tools release, Eos Trans $A G U$, 79, 329.

6) Yamamoto S., Noda S, and Korenaga M, 2012, An estimation method of epicentral distance based on characteristics of P-wave initial envelope, RTRI report, 26, 5-10 (in Japanese with English abstract).

7) Yoshimoto K, Sato H, and Ohtake M, 1997a, Three-component seismogram envelope synthesis randomly inhomogeneous semi-infinite media based on the single scattering approximation, Phys. Earth Planet Inter, 104, 37-61.

8) Yoshimoto K, Sato H, and Ohtake M, 1997b, Short-wavelength crustal heterogeneities in the Nikko area, Central Japan, revealed from the three-component seismogram envelope analysis, Phys. Earth Planet Inter., 104, 63-73. 